
\title{
25 Research Soure \\ Conversion of Chirality to Twisting via 1D-to-2D Growth of Graphene Spirals
}

\section{Zhu-Jun Wang ( $\nabla$ wangzhj3@shanghaitech.edu.cn )}

Shanghai Tech University

\section{Xiao Kong}

Institute for Basic Science

\section{Yuan Huang}

Beijing Institute of Technology https://orcid.org/0000-0002-7005-1319

\section{Lihong Bao}

Chinese Academy of Sciences https://orcid.org/0000-0002-2942-892X

\section{Kecheng Cao}

Shanghai Tech University, School of Physical Science and Technology

\section{Yuxiong Hu}

Shanghai Tech University, School of Physical Science and Technology

Jun Cai

Shanghai Tech University, School of Physical Science and Technology

\section{Lifen Wang}

Institute of Physics https://orcid.org/0000-0002-8468-5048

Jun Li

ShanghaiTech University

\section{Hui Chen}

Institute of Physics, Chinese Academy of Sciences https://orcid.org/0000-0002-3369-8113

\section{Fei Pang}

Renmin University of China

\section{Zhihai Cheng}

Beijing Key Laboratory of Optoelectronic Functional Materials \& Micro-nano Devices, Department of Physics

\section{Petr Bábor}

Brno University of Technology

\section{Miroslav Kolibal}

Brno University of Technology

\section{Zhongkai Liu}

ShanghaiTech University https://orcid.org/0000-0003-3373-8039

\section{Yulin Chen}

Shanghai Tech University, School of Physical Science and Technology 


\section{Qiang Zhang}

Tsinghua University https://orcid.org/0000-0002-3929-1541

\section{Yi Cui}

Chinese Academy of Sciences https://orcid.org/0000-0002-9182-9038

\section{Kaihui Liu}

State Key Laboratory for Mesoscopic Physics, Frontiers Science Center for Nano-optoelectronics, School of Physics, Peking University, Beijing 100871 https://orcid.org/0000-0002-8781-2495

\section{Haitao Yang}

Institute of Physics, Chinese Academy of Sciences https://orcid.org/0000-0003-4304-9835

\section{Xinhe Bao}

Dalian Institute of Chemical Physics

\section{Hong-Jun Gao}

Institute of Physics https://orcid.org/0000-0001-9323-1307

\section{Zhi Liu}

Shanghai Institute of Microsystem and Information Technology, Chinese Academy of Sciences

\section{Wei Ji}

Renmin University of China https://orcid.org/0000-0001-5249-6624

\section{Feng Ding}

Center for Multidimensional Carbon Materials, Institute for Basic Science (IBS); Ulsan National Institute of Science and Technology (UNIST) https://orcid.org/0000-0001-9153-9279

\section{Marc Willinger}

ETH Zurich

\section{Article}

\section{Keywords:}

Posted Date: February 9th, 2022

DOI: https://doi.org/10.21203/rs.3.rs-1302798/v1

License: (c) (1) This work is licensed under a Creative Commons Attribution 4.0 International License. Read Full License 


\section{Abstract}

The properties of two-dimensional (2D) van der Waals (vdW) materials can be tuned through nanostructuring or controlled layer stacking, where interlayer hybridization induces exotic electronic states and transport phenomena. Here, we describe an assisted self-assembly of twisted layer graphene. The process, which can be implemented in standard chemical vapour deposition (CVD) growth, is best described using the analogy to Origami and Kirigami of paper and involves wrinkle formation, folding, tearing, and re-growth. Inherent to the process is the formation of intertwined graphene spirals and conversion of the chiral angle of one-dimensional (1D) wrinkles into a 2D twist angle of a threedimensional (3D) superlattice. The approach can be extended to other foldable 2D materials and facilitates the production of miniaturized electronic components, including capacitors, resistors, inductors, and super-conductors.

\section{Main}

Growth techniques that enable the production of defect-free single-layer sheets of graphene and other 2D materials have been considerably developed in recent years ${ }^{1,2}$. The key requirement and remaining bottleneck for the realization of graphene-based nano-electronics lies in the ability to control the electronic properties of synthetic 2D structures, and to produce them in a reproducible and consistent manner at relevant manufacturing scales. Of specific interest is the tuneable interaction between electronic states and utilization of coupling phenomena that lead to exotic electronic properties in twisted layer stacks. As predicted by theory and experimentally demonstrated on a micro scale, semi-conducting, metallic, and even superconducting graphene layers can be obtained depending on the stacking angle ${ }^{3-6}$.

In this work, we summarize our systematic experimental and theoretical investigations on the growth of twisted-layer graphene. More specifically, we address the formation of graphene spirals (GSs), which are topological defects that connect individual graphene layers into a continuous, single-atom-thick helical structure that rotates around a screw dislocation axis, has been extensively studied ${ }^{7,8}$. While spiralled $s p^{2}$ carbon has been observed in annealed pyrolytic graphite and anthracite ${ }^{7,9,10}$, there have been no reports on mechanisms that allow for the controlled formation of such topological defects. Based on real-time spatially resolved observations of CVD graphene growth, we describe their formation and demonstrate how assisted self-assembly of intertwined spirals can be used to fabricate twisted layer graphene with a controllable twist-angle. We refer to the ancient art of paper folding, Origami, and cutting, Kirigami, and use simple paper models to describe the experimentally observed process. It basically consists of a sequence of wrinkle formation, folding, cutting and the subsequent growth of the twisted layer graphene $\mathrm{i}^{11-15}$. The process involves a novel conversion of the 1D chiral angle of folded wrinkles into a 3D twist angle between alternating stacked layers and thus, provides a key to direct control of the twist angle. Our experimental observations are supported by density functional theory (DFT) calculations and kinetic Monte-Carlo (KMC) growth simulations. The presented sequence of graphene Origami-Kirigami can be implemented in a standard CVD process and is, in principle, applicable for all foldable 2D 
materials. This work thus presents a blue-print for the production of miniaturized electronic components, including capacitors, resistors, inductor and super-conductors.

The here described mechanism for twisted layer formation was first revealed through direct observation of CVD growth that initiated under controlled conditions inside the chamber of a modified environmental scanning electron microscope $(E S E M)^{16}$. It relies on the ability to balance between graphene growth and etching, and facilitates temperature induced reconstruction of the substrate surface. In the following sections, the individual steps that are involved in assisted self-assembly of twisted layer graphene are presented.

\section{Formation of graphene wrinkles (Origami)}

The first step involves local formation of graphene wrinkles and is initiated directly after completing the CVD growth of a single graphene layer that conformally covers the surface of a polycrystalline platinum substrate. By heating the substrate beyond the initial annealing and subsequent graphene growth temperature $\left(\sim 1400^{\circ} \mathrm{C}\right)$, it is possible to re-induce abnormal grain growth ${ }^{17,18}$. During this process, high surface energy platinum grains with a pronounced stepped surface morphology can be converted into low-energy grains that expose flat surfaces ${ }^{17,19}$. As shown in the image sequence in Fig. 1a-1d, the reconstruction of a stepped into a flat surface takes place underneath the already grown single layer graphene via propagating grain-boundaries. The resulting reduction in surface area induces compressive strain in the graphene film ${ }^{19}$ and formation of wrinkles that are predominantly oriented parallel to the direction of the original step-edges ${ }^{13}, 20$. This process is schematically illustrated in Fig. 1e-1g. More details regarding the surface structure are available in Supplementary Fig. 1 and online movie 1.

\section{Wrinkle folding (Origami) and tearing (Kirigami)}

Once the formed graphene wrinkles reach a certain height, they get mechanically unstable due to the vdW attraction between the sidewalls. As a consequence, wrinkles collapse and fold towards the substrate ${ }^{21}$. This folding-over can happen to either of the two sides, as indicated in Fig. $\mathbf{1 g}, \mathbf{1} \mathbf{h}$ and visualized using paper Origami-Kirigami, as shown in Fig. 1i-1l. Folded wrinkles can be identified in the SEM images on the basis of the step-wise decrease of the secondary electron signal of each added graphene layer ${ }^{22}$. They form a narrow three-layer graphene ribbon with an $\mathrm{S}$ shaped cross-section and two tubular edges running along the folding line (of which one is buried, see illustration of the cross-section in in Fig $\mathbf{1 g}$ ). These tubular edges resemble structural features of single-walled carbon nanotubes, especially with respect to the high curvature at the folding edge and the defined chirality along the folding line. Due to the random nature of folding, the wrinkles have a chance to collapse towards opposite sides and thus create a node between them. The node then tears and the graphene wrinkles up, forming two pairs of open edges (blue arrows in Fig. $\mathbf{1 d}, \mathbf{1 g}, \mathbf{1 h}$ ). This process relates to the formation of GSs and will be discussed later.

\section{Cracking of tubular edges (Kirigami)}


In a well-adjusted CVD process, the attachment of carbon species at low-energy growth-fronts is accompanied by hydrogen etching at high-energy defective sites $^{23}$. It is thus possible to tune the balance between growth and etching, such that highly curved graphene at the exposed tubular edge of a folded wrinkle is selectively etched (see Extended Data Fig. 2 for details). This generates two new growth fronts: one associated to the top sheet (highlighted by the green lines in Fig. 1h, 19), and one associated with the backwards-folded bottom sheet (see Origami-Kirigami illustration in Figure $\mathbf{1} \mathbf{g}$-h and experimental observation in Figure $1 \mathbf{c}-\mathbf{d}$ ). The attachment of carbon species at these newly formed graphene edges leads to the development of low-energy zig-zag edges growing on top of the original layer (see the sawtooth shape in the yellow square in Fig. $\mathbf{1 d}$ and the green and pale blue lines in Fig. $\mathbf{~ h}$, and Extended Data Fig. 3). Wrinkle folding, tearing, cracking and re-growth thus results in the formation of twisted trilayer graphene, and the whole process is illustrated using an analogy of Origami-Kirigami in Fig. 1i-1 ${ }^{13}$, 24 .

\section{Formation of GSs}

As mentioned above, wrinkles can fold towards either of the two sides. It is therefore possible that wrinkles tear up into segments that are folded towards alternating sides. The tearing of wrinkles (analogous to Kirigami) gives rise to additional growth fronts, as illustrated in Fig. 1l using a paper model. The attachment of carbon species to these new growth fronts leads to the formation of a pair of intertwined double-layer spirals that are rotating around a common screw dislocation axis. Experimental evidence for the occurrence of such processes is presented in Fig. 2a-2d, and in agreement with kinetic Monte Carlo (KMC) based growth simulations, as shown in Fig. 2e-2h (see Supplementary Note for details and online movie 2).

The resulting GS can be viewed as a screw dislocation formed by four graphene layers, of which two originate at each tearing edge (see also Extended Data Fig. 4, 5). As a consequence of the opposite folding direction of collapsed wrinkles, the tearing edges at the nodal points always have reversed handedness to those at the two adjacent nodal points (refer to Extended Data Fig. 4). Moreover, the spirals grow upwards, as confirmed using post-growth scanning probe microscopy imaging (Extended Data Fig. $\mathbf{5 , 6})^{25}$. Therefore, the handedness of GSs can be determined by in-plane observation, as highlighted in Fig. 2b-2d.

It is worth noting that the starting and end-points of a graphene wrinkle have the shape of a half cone (Extended Data Fig. 7a-7c) ${ }^{26,27}$. The rupture of the tubular edge up to the apex of a wrinkle creates an endpoint at which subsequent growth can originate (shown using the Origami in Extended Data Fig. 7d7f 26,27 . Thus, the two growth fronts formed at the cracked tubular edge are forced into a spiral growth, with spirals winding around the tail end of the wrinkle, as shown in Extended Data Fig. $\mathbf{7 g}$ and in the Origami model (see colored arrows in Extended Data Fig. 7f, 7i and 7k). Therefore, the GS at end of a graphene wrinkle can be viewed as a screw dislocation formed by two graphene layers.

1D chirality to 2D twist-angle conversion in the graphene spiral development 
The STM image recorded on a GS shows Moiré superstructures formed by the two topmost graphene layers rotating around a fixed axis (Extended Data Fig. 6b). In contrast to classical screw-dislocation based spirals, where their in-plane layer orientation is not affected ${ }^{28,29}$, the GSs are initiated from the chirality of the wrinkle.

We used paper with printed graphene lattices to model the graphene layer and to explain how a 1D chiral angle was converted to the twisting angle of a graphene spiral (Fig. 2j-2l and Extended Data Fig. 8). In this model, the chiral angle, $\theta$, represents the angle of a wrinkle relative to the zigzag direction of the graphene lattice (Fig. 2j). A flattened graphene wrinkle (Fig. 2l) contains two graphene layers (Extended Data Fig. $\mathbf{8 e}, \mathbf{2 h}$ ), where the top layer has the exact same lattice orientation as the graphene basal plane, but the bottom layer has a twist angle of $2 \theta$ relative to the basal plane. During the folding-cracking process, the chiral angle of the graphene wrinkle was thus increased to two times that of the twisting angle between the two graphene layers. In this case, the GSs forming at the tearing points result in a twisting angle between the adjacent layers being out-of-plane. Therefore, the transfer from the chirality of a graphene wrinkle to the twist angles of stacked graphene layers lays the foundations for designing the pattern of twists in multilayer graphene along the out-of-plane direction.

As shown in Fig. $2 m-2 p$, in the case of the two graphene folds on both sides of a nodal point with the same chirality, $\theta$, the twisting angle pattern of the graphene spiral will be in the sequence of $2 \theta / 0 / 2 \theta / 0 /$. In the case of the two graphene folds not being parallel (Fig. 2q-2t), the twisting angle pattern of the GS will be $2 \theta_{1} / 0 / 2 \theta_{2} / 0 /$, where $\theta_{1}$ and $\theta_{2}$ are the chirality of the two graphene folds on both sides of the node, respectively. Both cases can be clearly seen experimentally (Fig 2a-2c).

\section{The coalescence of graphene layers between two neighbouring spirals}

As the graphene layers around a GS grow, the layers from neighbouring spirals will meet and coalesce. The further evolution of GSs (Fig. 3a-3c and online movie 3) was directly imaged by in situ ESEM, where the real-time and real-space details of coalescence of the graphene layers with neighbouring GSs can be clearly seen (Fig. 3d-3e). In the case of overlapped graphene layers, the images display stepwise contrast variations in the merging region (Extended Data Fig. 9) ${ }^{28}$. However, the contrast between individual layers is homogenous in the merging region of the two spirals, which indicates layer-by-layer coalescence with neighbouring GSs. When two oppositely rotated growth fronts coalesce from two neighbouring GSs, their opposing Burgers vectors annihilate, as expected for Frank-Read sources ${ }^{30}$; this therefore demonstrates the layer-by-layer manner to the growth of GSs.

The coalescence of each corresponding layer with the two intertwined twisted spirals is seamless, as indicated by the appearance of rounded concave corners with edges at an angle of $19^{\circ} 31$ (highlighted by the orange arrow in Fig. 3e and Extended Data Fig. 9). This observation is favoured from an energetic point of view ${ }^{32}$. The seamlessness is ascribed to the oppositely rotated screw dislocations originating on the same wrinkle with the same basal plane. The coalescence of GSs becomes more complicated if the folding axis bend across the tearing nodal point, which is further described in Extended Data Fig. 10. 
In order to experimentally verify the occurrence of the conversion from 1D chirality to 2D twisting angle, we need to first identify the orientation of the wrinkle axis with respect to the graphene lattice. This can be done by comparing the wrinkle axis with the growth fronts of the graphene basal layer.

While the zigzagged edges of the basal layer are highlighted by the blue lines in Fig. 3a, the folding-over axis (the red line) of the wrinkle (the dark ribbon) appearing in Fig. $3 \mathbf{b}$ is tilted by approximately $7^{\circ}$ with respect to the zigzag direction (the blue line). This $\sim 7^{\circ}$ tilting (chirality) angle should, as exemplified in the Origami-Kirigami model (Fig. 2j-2l and Extended Data Fig. 6), double to $\sim 14{ }^{\circ}$ for the twisting angle between the top and bottom layers of the folded-over wrinkle. This was detectable even at the meso-scale (Fig. 3f).

Fig. $\mathbf{3 g}$ and $\mathbf{3 h}$ show TEM images of, at least six layers, GSs exfoliated from the sample grown inside the ESEM chamber (the details are referred to in Extended Data Fig. 11). Both the differently coloured hexagons in the selected area electron diffraction (SAED) patterns (Fig. $\mathbf{3 g}$ ) and the fast Fourier transform (FFT) patterns of the atomic-resolution image (Fig. 3h) highlights the twisting angle of $\sim 14^{\circ}$ at the nano- and atomic-scales, respectively, which is consistent with the in situ ESEM observations conducted at the meso-scale.

Our KMC simulations (Fig. 3i-3k) successfully reproduced the experimentally observed coalescence of the GSs and the Moiré pattern (refer to Extended Data Fig. 12 and online movie 3), i.e. the chirality of the $7^{\circ}$ and $14^{\circ}$ twisting angle. The slightly different shape of the $2^{\text {nd }}$ layer between experimental observation and simulation was caused by the polygonal domain (Extended Data Fig. 13). This striking consistency between the experimental observation and theoretical modelling further validates our proposed mechanism of the $1 \mathrm{D}$ chirality to $2 \mathrm{D}$ twist-angle conversion.

It is important to note that the multi-layer graphene grown by the coalescence of GSs shows a clear layerby-layer manner of growth (Frank-van der Merwe growth) because the opposite Burgers vectors of the two spirals cancel each other out on the whole. Ignoring the central area of the multilayer graphene, where two graphene spiral appears, the stacking order of the multilayer graphene is exactly same as that grown via layer-by-layer nucleation. This explains the broadly observed multilayer graphene growth in many CVD processes, where the multilayer region is generally very thick and the twist angles are well defined ${ }^{22,33,34}$.

\section{Multi-layer graphene growth initiated by multiple GSs}

The presented mechanism of GS formation can be tested against experimental results and used to reverse derive the structure of multilayer graphene. Extended Data Fig. 14a shows a complex vertical stacking order which has previously been interpreted as $30^{\circ}-30^{\circ}$ rotation between successive layers 22 . Closer inspection illustrates that the stack consists of alternating polycrystalline and single crystalline layers. The polycrystalline layers can be identified by the concave corners that are highlighted by white arrows in Extended Data Fig. 14a ${ }^{35}$. Identical shapes are regularly repeated, in one-layer intervals, with alternating odd and even numbered layers. The odd numbered layer shows a hexagonal intrinsic shape of graphene, while the even numbered layer appears as a specific concave polygonal shape, highlighted 
using different coloured lines in Extended Data Fig. 14a. This oscillatory behaviour in the shapes of the even and odd numbered layers in multi-layer graphene hints to the fact that the odd/even layers have some connection and result in a similar shape. The strength of the graphene-substrate interaction cannot affect layer growth in the out-of-plane direction, with a distinction between even-odd layer number. Thus, the only explanation is that the multi-layer stacking structure is essentially due to the coalescence of GSs. The shape of the even-odd layer provides clues to reverse derive the orientation of spirals and their initiation. As addressed in Extended Data Fig. 10v, the concave polygonal even layers imply polycrystalline film, and are thus attributed to coalescence of misaligned spirals with opposite handedness along the same glide plane ${ }^{35}$, while the hexagonal odd layers result from coalescence of aligned spirals with opposite handedness. Based on the shapes of the even-odd layers and the fundamental principle of screw dislocation initialization from graphene wrinkles (refer to Extended Data Fig. 10), the spiral orientation, rotation and Burger vector can be estimated. While the nodal point layout, containing five nodal points demonstrated in Extended Data Fig. 14b-d, is the minimal set of spirals required to reproduce the final shape, additional spiral cores aligned in parallel do not change the final shape of the spiral crystal. To rationalize reverse inference of the GS structure (Extended Data Fig. 14b, c), we simulated the shape evolution of GSs using conjectural nodal point layout (see Extended Data Fig. 14d). The evolution of GSs during growth was simulated by KMC, starting from a cracked and teared folded-over wrinkle (refer to Extended Data Fig. 15). The simulated coalescence shape of GSs (Extended Data Fig. 14e-h) successfully recreate the experimentally observed shapes, providing further validation on the rationalities and efficiencies of the theoretical model.

\section{Macroscopic crystals and properties}

The transport measurements of the as-grown GSs were conducted to evaluate the electronic properties. Fig. 4a shows an atomic force microscopy (AFM) image of a GS sample. To measure the transport properties along the spiral axis direction, we fabricated a measurement setup as shown in both the optical microscope and SEM images in Fig. $\mathbf{4 c}$ and $\mathbf{4 d}$, respectively. Fig. $\mathbf{4 b}$ depicts a schematic of the measurement setup, where the applied current and magnetic field are along the spiral direction. We also measured a highly ordered pyrolytic graphite (HOPG) sample with the same thickness for comparing. Fig. $4 \mathrm{e}$ plots the Z-axis magnetoresistances of both samples at $20 \mathrm{~K}-2 \mathrm{~K}$. The spiral exhibits a Z-axis magnetoresistance of roughly $2 / 3$ that of the HOPG and a semi-metallic behaviour, which is, most likely, due to the continuously rotating graphene layers in the GS sample that band transport rather than interlayer tunnelling in the HOPG sample, which plays a dominant role in the GS. For the magnetoresistance of the GS, a linear behaviour is observed at the high-field region (Fig. $\mathbf{4 f}$ ), which is different to that of the HOPG (Fig. $\mathbf{4 g}$ ). This should therefore be the required subject of future investigations.

\section{Conclusion And Outlook}

In summary, we herein report a graphene Origami-Kirigami approach (wrinkling, folding, tearing and cracking) that leads to spiral growth of graphene multilayers with controlled stacking orders. The 
coalescence of the graphene multilayers originated from neighbouring graphene spirals and lead to the Frank-van der Merwe growth of graphene. This process converts the chirality of a one-dimensional graphene wrinkle into the twist angles of the two-dimensional stacking layers of graphene. The kinetic pathways are observed by real-time imaging and rationalized, and the abstracted mechanism for chirality conversion is validated by theory-based simulations of spiral evolution. Thus, the twisted stacked graphene layers with small misalignment between adjacent layers are tuneable by varying the chiral graphene wrinkle, and are of interest due to unconventional superconductivity ${ }^{6}$. This work has profound implications in the development of future nano-inductance devices and provides a step towards the scalable fabrication of multi-layered graphene with defined twist angles.

Overall, this Origami-Kirigami approach resulted in 1D to 2D angle conversion and allowed us to engineer the twisting angles in multi-layered graphene, which is, in principle, applicable for all foldable 2D materials. Our study therefore offers a general method to convert the chirality of one-dimensional wrinkles into the twist angle of vertically stacked 3D superlattices.

\section{Declarations}

\section{Contributions}

Z.-J.W. conceive of this project and supervised the research; Z.-J.W. and M.-G.W. modified the ESEM; Z.J.W. planned and conducted the in situ growth experiments; Z.-J.W. did most of the ESEM data analysis; Z.-J.W., K.C., Y.H. and L.W. performed TEM and AFM measurements; theoretical simulations and implementation of the obtained results was done by X.K. and F.D.; STM measurements were performed by Y.C., H.C., H.-J.G. and X.B.; L.B. and J.L. performed transport measurements. important contributions to the interpretation of the results, conception and writing of the manuscript were made by Z.-J.W., W.J, F.D. and M.-G.W. All authors participated in the scientific discussion.

\section{References}

1. $\mathrm{Xu}, \mathrm{X}$. et al. Ultrafast epitaxial growth of metre-sized single-crystal graphene on industrial Cu foil. Sci. Bull. 62, 1074-1080 (2017).

2. Wang, L. et al. Epitaxial growth of a 100-square-centimetre single-crystal hexagonal boron nitride monolayer on copper. Nature 570, 91-95 (2019).

3. Trambly de Laissardière, G., Mayou, D. \& Magaud, L. Localization of Dirac Electrons in Rotated Graphene Bilayers. Nano Lett. 10, 804-808 (2010).

4. Suárez Morell, E., Correa, J.D., Vargas, P., Pacheco, M. \& Barticevic, Z. Flat bands in slightly twisted bilayer graphene: Tight-binding calculations. Phys. Rev. B 82, 121407 (2010). 
5. Kim, K. et al. Tunable moire bands and strong correlations in small-twist-angle bilayer graphene. Proc. Natl. Acad. Sci. U.S.A. 114, 3364-3369 (2017).

6. Cao, Y. et al. Unconventional superconductivity in magic-angle graphene superlattices. Nature 556, 43-50 (2018).

7. Hennig, G.R. Screw Dislocations in Graphite. Science 147, 733-734 (1965).

8. $\mathrm{Xu}, \mathrm{F} ., \mathrm{Yu}, \mathrm{H}$., Sadrzadeh, A. \& Yakobson, B.I. Riemann Surfaces of Carbon as Graphene Nanosolenoids. Nano Lett. 16, 34-39 (2016).

9. Patel, A.R. \& Bahl, O.P. Evidence of screw dislocations in graphite. Br. J. Appl. Phys. 16, 169-171 (1965).

10. Sun, Y.Q., Alemany, L.B., Billups, W.E., Lu, J.X. \& Yakobson, B.I. Structural Dislocations in Anthracite. J. Phys. Chem. Lett. 2, 2521-2524 (2011).

11. Allen, M.J. et al. Chemically induced folding of single and bilayer graphene. Chem. Commun., 6285-6287 (2009).

12. Mu, J.K. et al. Origami-inspired active graphene-based paper for programmable instant self-folding walking devices. Sci. Adv. 1, 8 (2015).

13. Ebbesen, T.W. \& Hiura, H. Graphene in 3-dimensions: Towards graphite origami. Adv. Mater. 7, 582586 (1995).

14. Blees, M.K. et al. Graphene kirigami. Nature 524, 204-207 (2015).

15. Miskin, M.Z. et al. Graphene-based bimorphs for micron-sized, autonomous origami machines. Proc. Natl. Acad. Sci. U. S. A. 115, 466-470 (2018).

16. Wang, Z.-J. et al. Direct Observation of Graphene Growth and Associated Copper Substrate Dynamics by in Situ Scanning Electron Microscopy. ACS Nano 9, 1506-1519 (2015).

17. Hillert, M. On the theory of normal and abnormal grain growth. Acta Metall. 13, 227-238 (1965).

18. Wu, M. et al. Seeded growth of large single-crystal copper foils with high-index facets. Nature 581, 406-410 (2020).

19. Jin, S. et al. Colossal grain growth yields single-crystal metal foils by contact-free annealing. Science 362, 1021-1025 (2018).

20. Sutter, P.W., Flege, J.-I. \& Sutter, E.A. Epitaxial graphene on ruthenium. Nat. Mater. 7, 406-411 (2008). 
21. Zhu, W. et al. Structure and Electronic Transport in Graphene Wrinkles. Nano Lett. 12, 3431-3436 (2012).

22. Wang, Z.-J. et al. Stacking sequence and interlayer coupling in few-layer graphene revealed by in situ imaging. Nat. Commun. 7, 13256 (2016).

23. Yang, R. et al. An Anisotropic Etching Effect in the Graphene Basal Plane. Adv. Mater. 22, 40144019 (2010).

24. Chen, $\mathrm{H}$. et al. Atomically precise, custom-design origami graphene nanostructures. Science $\mathbf{3 6 5}$, 1036-1040 (2019).

25. Zhao, Y. et al. Supertwisted spirals of layered materials enabled by growth on non-Euclidean

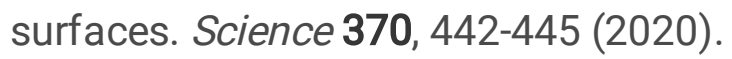

26. Zhang, K. \& Arroyo, M. Understanding and strain-engineering wrinkle networks in supported graphene through simulations. J. Mech. Phys. Solids 72, 61-74 (2014).

27. Pereira, V.M., Castro Neto, A.H., Liang, H.Y. \& Mahadevan, L. Geometry, mechanics, and electronics of singular structures and wrinkles in graphene. Phys. Rev. Lett. 105, 156603 (2010).

28. Tay, R.Y. et al. Concentric and Spiral Few-Layer Graphene: Growth Driven by Interfacial Nucleation vs Screw Dislocation. Chem. Mater. 30, 6858-6866 (2018).

29. Rakovan, J. \& Jaszczak, J.A. Multiple length scale growth spirals on metamorphic graphite $\{001\}$ surfaces studied by atomic force microscopy. Am. Miner. 87, 17-24 (2002).

30. Frank, F.C. \& Read, W.T. Multiplication Processes for Slow Moving Dislocations. Phys. Rev. 79, 722723 (1950).

31. Wang, Z.J. et al. The Coalescence Behavior of Two-Dimensional Materials Revealed by Multiscale In Situ Imaging during Chemical Vapor Deposition Growth. ACS Nano 14, 1902-1918 (2020).

32. Nabarro, F.R.N. The force between two screw dislocations. Philos. Mag. A 54, 577-582 (1986).

33. Karamat, S. et al. Coalescence of few layer graphene grains grown by chemical vapor deposition and their stacking sequence. J. Mater. Res. 31, 46-54 (2016).

34. Sun, J. et al. Growth mechanism of graphene on platinum: Surface catalysis and carbon segregation. Appl. Phys. Lett. 104 (2014).

35. Guo, W. et al. Governing Rule for Dynamic Formation of Grain Boundaries in Grown Graphene. ACS Nano 9, 5792-5798 (2015). 


\section{Methods}

In situ observations by environmental SEM. In situ CVD growth experiments were performed inside the chamber of a modified commercial ESEM (Thermo Fisher Quattro ESEM). The vacuum system of the ESEM was modified and upgraded with oil-free pre-vacuum pumps. The instrument is equipped with a homemade laser heating stage, a gas supply unit (mass flow controllers from Bronkhorst), and a mass spectrometer (Pfeiffer OmniStar) for the analysis of the chamber atmosphere. Owing to the use of rubber O-rings for the sealing and the fact that the chamber cannot be baked out, the base pressure of the instrument is around $2 \times 10^{-5} \mathrm{~Pa}$, with a residual gas composition mostly comprising water, $\mathrm{N}_{2}$, and $\mathrm{O}_{2}$. After each sample loading, the chamber was pumped out to around $10^{-3} \mathrm{~Pa}$, purged with pure nitrogen, and pumped again to $10^{-3} \mathrm{~Pa}$ successively for several times. Under CVD growth conditions, the pressure is 6 orders of magnitude higher than the base pressure and constitutes mostly $\mathrm{H}_{2}(99.9995 \%$ purity) and $\mathrm{C}_{2} \mathrm{H}_{4}(99.95 \%$ purity). Samples of sizes ranging from $4 \times 4$ to $5 \times 5 \mathrm{~mm}$ are extracted from a $0.2 \mathrm{~mm}$ thick Pt foil (99.999\% purity). Prior to all CVD growth experiments, the chamber of the ESEM was plasma cleaned. The Pt foil was annealed at $1000^{\circ} \mathrm{C}$ under a hydrogen flow of $10 \mathrm{sccm}$ at $25 \mathrm{~Pa}$ for more than $48 \mathrm{~h}$ inside the chamber. The temperature was measured via a B-type thermocouple that was spot-welded onto the substrate, which simultaneously served to ground the sample. The CVD growth was performed at temperatures ranging from $1000^{\circ} \mathrm{C}$ to $1400{ }^{\circ} \mathrm{C}$, with a pressure in the chamber ranging from $25 \mathrm{~Pa}$ to $200 \mathrm{~Pa}$. During the experiments, the microscope was operated at an acceleration voltage of 3.0-7.5 kV. Images were recorded by a large field detector during CVD growth conditions. No influence of the electron beam on the growth and etching process could be observed. The imaged regions and their respective surroundings showed similar behaviour, as evidenced by changing the magnification or by moving the sample under the beam. Furthermore, no electron beam induced contamination was observed at elevated temperatures.

Electron Backscatter Diffraction (EBSD). The EBSD patterns were acquired using the EDAX Digiview detector installed on a SEM FEI NovaNano SEM 230. Analysis of EBSD patterns, including phase identification and generation of orientation maps, was performed with the EDAX OMI 5.31 program.

Scanning tunnelling microscopy (STM). STM measurements were performed at room temperature in an UHV system with base pressure in the range of $10^{-8} \mathrm{~Pa}$, equipped with STM, LEED apparatus, $\mathrm{Ar}^{+}$ sputtering gun, laser heating stage, and gas feeding system. The images were recorded with a constant current mode using a homemade W-tip.

Transmission Electron Microscopy (TEM). TEM experiments were performed in FEl Titan Themis G2 300 operated at $80 \mathrm{kV}$.

Atomic Force Microscopy (AFM). AFM images were recorded on a Bruker Sharp Nitride Lever probe (SNL10). Imaging was done in tapping mode using a V-shaped cantilever probe B (silicon-tip on Nitride Lever with frequency $\mathrm{f} 0=40-75 \mathrm{kHz}$ and spring constants $\mathrm{k}=0.32 \mathrm{Nm}$ ). 
Transport measurements. Hall-bar electrodes on $\mathrm{Si} / \mathrm{SiO}_{2}$ substrate were fabricated by a standard photolithography technique, followed by electron beam evaporation of $\mathrm{Ti} / \mathrm{Au}(5 / 60 \mathrm{~nm})$ electrodes. The graphene spiral was transferred onto the electrodes in a glovebox, and covered by BN for insulating, and then a multi-layer graphene was loaded onto the graphene spiral as a top lead. Thus, the current can flow through the Z-axis of the graphene spiral, and also the exact Z-axis resistance can be observed as illustrated in Fig. 4b. Transport measurements were performed in a Physical Property Measurement System (PPMS, Quantum Design Inc.) cryostat. A constant $1 \mu \mathrm{A}$ a.c. current above $10 \mathrm{~Hz}$ was applied for all the magneto-transport measurements.

\section{Figures}

\section{Figure 1}

Observation and illustration of graphene Origami and Kirigami. (a-d) In situ ESEM images recorded during a high-temperature annealing step $\left(1400^{\circ} \mathrm{C}, 25 \mathrm{~Pa}, \mathrm{H}_{2}: \mathrm{C}_{2} \mathrm{H}_{4} \sim 100: 1\right)$ showing the migration of a Pt grain boundary underneath an already grown graphene layer (red arrows). (e-h) schematic illustrations depicting the process in top (upper panels) and cross-section (lower panels) view. (i-l) paper model showing wrinkle formation induced by the surface reconstruction of the substrate, as well as wrinkle folding and rapture into segments folded to different sides $(k, l)$. The number of stacked graphene layers in the SEM images is apparent from the lowering of image brightness for each added layer as shown in the grey-level scale in (d) and reproduced in (e-h). The Pt grain boundaries are highlighted by red dashed lines in panels, while the red arrows indicate the direction of the propagating boundary. Purple solid lines denote exposed tubular edges, while the purple arrows mark the location of the edges in panels $\mathrm{c}, \mathrm{g}$, and $k$. Blue arrows show the tearing edges in panels $d, g, k$ and $\mathrm{l}$. Etching and re-growth along the exposed tubular edge results in extension of the $2^{\text {nd }}$ and $3^{\text {rd }}$ layer as indicated in (h) by light blue and green dashed lines and arrows, respectively. The side-views in panels e-h represent line cuts at the locations highlighted by the yellow lines in their corresponding top-views. A series of zoomed-in images of the area highlighted by the yellow rectangle in panel d is shown in Extended Data Fig. 3.

\section{Figure 2}

Initialization of spiral growth at teared wrinkles. In situ SEM images of graphene spirals with opposite screw dislocation handedness at increasing magnifications (a-d). A large area image (a) shows a chain comprised of pairs of oppositely rotated spirals, where a tri-spiral region and a twin spiral region are magnified in (b) and (c), respectively. KMC simulations (e-h) reproduce the growth process of the tri-spiral shown in (b), in which the tearing nodal points were marked by red crosses and the left- and righthandednesses were indicated using green and red curved arrows in (b-d), (f), (k-m) and (q), respectively. 
Inset of panel e shows an analogous paper model of three oppositely rotated tearing points. Reasoning of the double rotation angle after tearing and cracking is illustrated in (i-l). An atomically resolved STM image (i) of a graphene wrinkle identifies its chiral angle, $\theta$, off the zigzag direction, in which the wrinkle axis is along the red line, and the blue arrow indicates the zigzag direction, as illustrated in the paper model within (j). After subsequent folding, tearing, and cracking, the wrinkle forms two nodal points with opposite chirality $(\mathrm{K}, \mathrm{I})$ and the $2^{\text {nd }}$ cracking edge is $2 \theta$ along the zigzag direction $(\mathrm{I})$. KMC simulations of the spiral growth processes $(\mathrm{m}-\mathrm{t})$ initialized from a folded, torn, and cracked nodal point in the presence of neighbouring folds with the same $(m-p)$ and differential $(q-t)$ chiral angles.

\section{Figure 3}

Growth and coalescence of graphene spirals. (a-f) In situ ESEM images of pairwise GS evolution with opposite screw dislocation handedness at different growing times, showing the chirality conversion from a 1D wrinkle to a twist angle of 3D stacking layers. The blue lines and arrows indicate the zigzagterminated edges and the corresponding direction, respectively. The wrinkle axis is along the red line in (b). ( $g$ ) is the TEM image of the GSs observed in (a-f) after coalescence inside the ESEM chamber and the corresponding SAED pattern highlighted by yellow and purple circles. (h) Atomically resolved graphene GB [magnification of the red circle in (g)] and the inset shows its fast Fourier transform (FFT) pattern. The corresponding seamless coalescence process of the GS pair is simulated using KMC and presented in (ik). The handedness is indicated by the red (right-handedness) and green (left-handedness) curved arrows (the arrow gives the direction of advance along the Z-axis). 


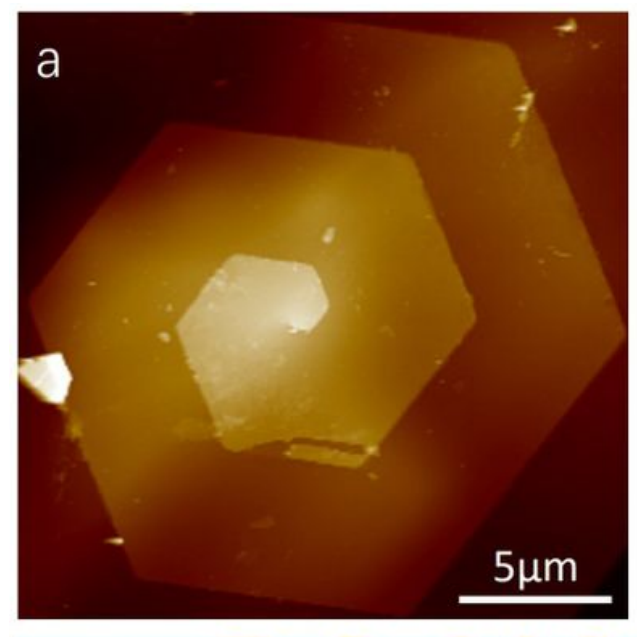

b
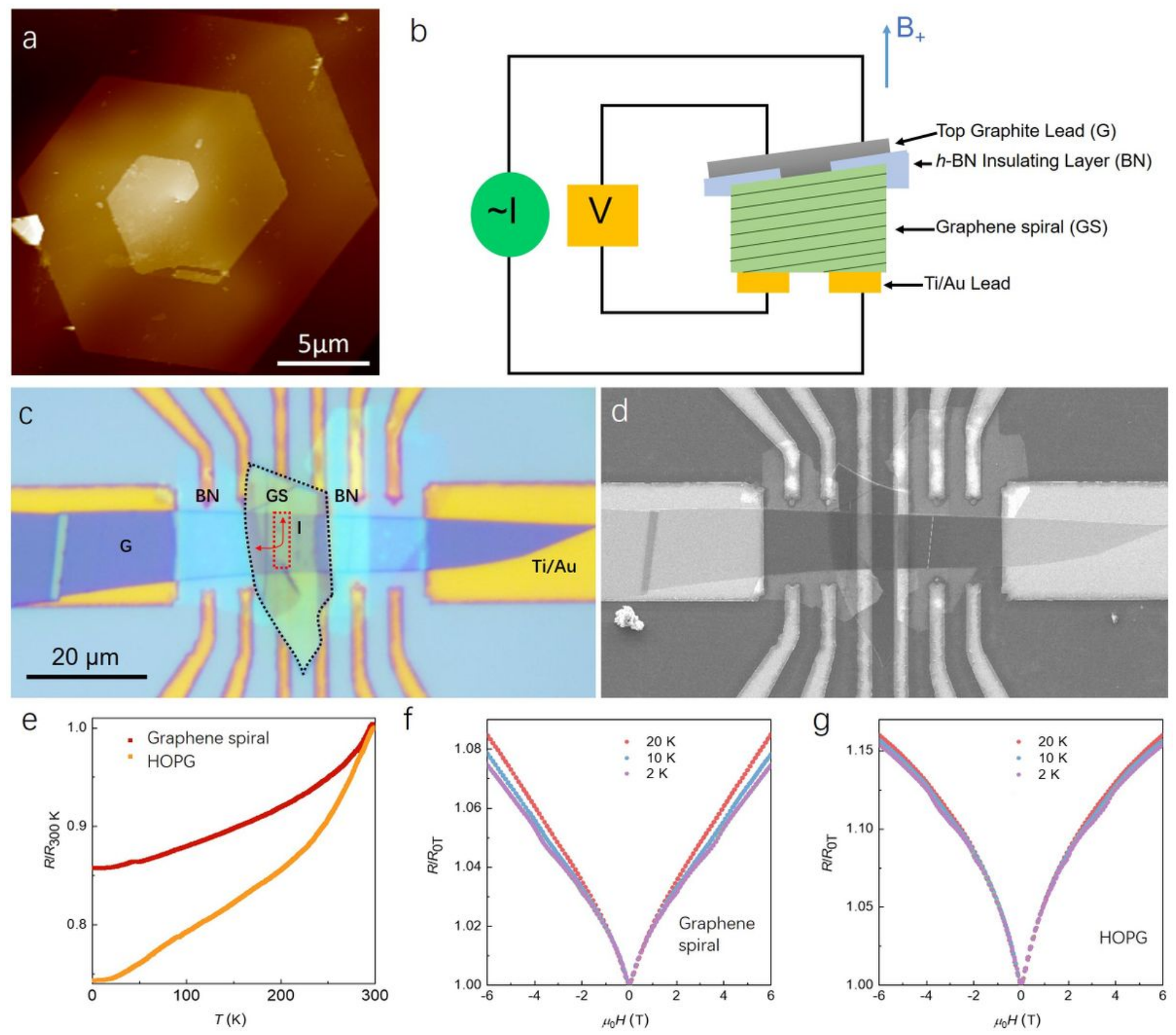

\section{Figure 4}

Comparison of transport properties between GS and HOPG. (a) Atomic force microscope image of a GS sample showing the exact centre of the GS. (b) Schematic image illustrating the setup for the transport property measurements. Here, electrical current flowing through the out-of-plane direction of the sample was recorded under a magnetic field applied along the same direction. Optical (c) and SEM (d) images show the top-view for the measurement setup. (e) Temperature dependence of normalized resistances $R / R_{300 \mathrm{~K}}$ for GS (red) and HOPG (orange), where the GS sample always shows a larger vertical resistance. Normalized magnetoresistances $R / R_{0 T}$ for GS (f) and HOPG (g) at $2 \mathrm{~K}$ (violet), $10 \mathrm{~K}$ (blue) and $20 \mathrm{~K}$ (red), respectively. 


\section{Supplementary Files}

This is a list of supplementary files associated with this preprint. Click to download.

- GrapheneSpiralSupplementaryInformation.docx

- OnlineMovie1.avi

- OnlineMovie2.avi

- OnlineMovie3.avi 